\title{
APPLICATION OF ANISOTROPIC TEXTURE COMPONENTS
}

\author{
TH. ESCHNER* and J.-J. FUNDENBERGER \\ Université de Metz. ISGMP/LM2P, Ile du Saulcy, F-57045 Metz Cedex 1
}

(Received 18 April 1996)

The description of textures in terms of texture components is an established conception in quantitative texture analysis. Recent developments lead to the representation of orientation distribution functions as a weighted sum of model functions, each corresponding to one anisotropic texture component. As was shown previously, an adequate texture description is possible with only a very small number of anisotropic texture components. As a result, textures and texture changes can be described by a small number of vivid parameters and their variations, namely by volume parts, half widths and ideal orientations.

The texture of a tensile tested commercial aluminum alloy was investigated by decomposition into anisotropic components. The texture evolution during tensile testing is represented by the corresponding changes of the component parameters and compared with results from an iterative series expansion analysis.

KEY WORDS: Quantitative texture analysis, anisotropic texture components, model functions, AA5182, tensile testing.

\section{INTRODUCTION}

Quantitative texture analysis is based on the description of relative crystal orientation frequencies in terms of a non-negative probability density called the orientation distribution function (ODF) $\left(V_{0}\right.$ - sample volume)

$$
f(g) d g=\frac{1}{8 \pi^{2}} \frac{d V(g)}{V_{0}} .
$$

As a rule, the ODF is calculated from experimentally determined pole figures by one of a various number of pole figure inversion methods, the series expansion method being the most widely used among them.

It is common practice to characterize a certain orientation distribution function by its maxima in distinct points in Euler-space, associating with each maximum one texture component, i.e. some volume fraction of crystallites with crystal lattice orientations near to the orientation at the point of maximum. Although currently there is no strong and complete physical theory of texture component formation this approach has proven to be suitable at least for the description of textures and texture changes.

* Present address: Max-Planck-Institut für Metallforschung, Pulvermetallurgisches Laboratorium, Heisenbergstraße 5, D-70569 Stuttgart. 
A more quantitative description of textures in terms of texture components became available after the introduction of Gauss-type model functions (Bunge, 1969) and so called standard distributions (Matthies, 1980), the latter providing a new way to solve the pole figure inversion problem (Helming, Eschner, 1990). The drawback of all these functions describing only isotropic texture components is in the need to approximate anisotropic ODF maxima by several closely arranged isotropic texture components. As a result, several types of model functions for anisotropic texture components were developed, e.g. (Savjolova, 1987), (Dnieprenko, Divinskii, 1993), (Eschner, 1993). The most promising model function from a practical point of view is described in some detail in the next section.

\section{ANISOTROPIC TEXTURE COMPONENTS}

A generalized model function suitable for the description of anisotropic texture components may be obtained from the standard distribution (Matthies, 1980)

$$
f\left(g, g_{0}\right)=f\left(g_{0}^{-1} g\right) \sim \exp (S \cos \omega)
$$

by making the constant half width parameter $S$ a variable of $g$ - the current position in Euler-space and $g_{0}$ - the point in Euler-space where the function reaches its maximum. $\omega$ is the angular distance between $g$ and $g_{0}$.

By representing the orientation distance $g_{0}^{-1} g$ by the angular distance and a rotation vector $g_{0}^{-1} g=[\omega, \vec{n}]$ one can choose $S$ to be $S=1 /|\vec{r}|^{2}$ with $\vec{r}$ being a vector parallel to $\vec{n}$ and pointing from the center of an ellipsoid to its surface (see Figure 1). The orientation of the ellipsoid's principal axes $A, B$ and $C$ with respect to the sample coordinate system is given by an auxiliary rotation $g_{d}$.

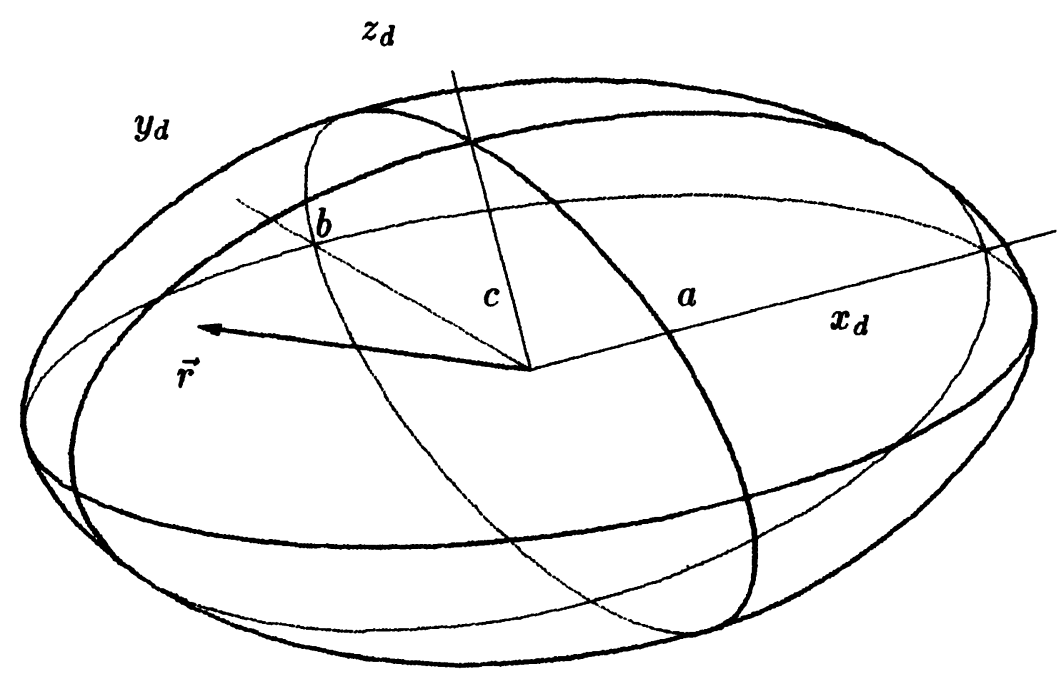

Figure 1 Definition of a variable half width parameter $S=1 /|\vec{r}|^{2}, \vec{r} \| \vec{n}$ with the help of an ellipsoid rotated with respect to the sample coordinate system by $g_{d}$. 
For that choice of $S$, analytical expressions for ODF and pole figure values can be found (for details, see (Eschner, 1994)):

$$
\begin{aligned}
& A=1 / a \\
& B=1 / b \\
& C=1 / c \\
& \alpha_{1}=\frac{1}{2}\left(-A^{2}+B^{2}+C^{2}\right) \\
& \alpha_{2}=\frac{1}{2}\left(A^{2}-B^{2}+C^{2}\right) \\
& \alpha_{3}=\frac{1}{2}\left(A^{2}+B^{2}-C^{2}\right) \\
& f\left(g, g_{d}, g_{0}, A, B, C\right)=N(A, B, C) \exp \left(\alpha_{1} A_{11}+\alpha_{2} A_{22}+\alpha_{3} A_{33}\right) \\
& \left\|A_{i j}\right\|=g_{d}^{-1} g g_{0}^{-1} g_{d} \\
& g_{d} \vec{h}=\left(h_{x}^{*}, h_{y}^{*}, h_{z}^{*}\right) \\
& g_{d}^{-1} g_{0} \vec{y}=\left(y_{x}^{+}, y_{y}^{+}, y_{z}^{+}\right) \\
& x=\alpha_{1} y_{x}{ }^{+} h_{x}{ }^{*}+\alpha_{2} y_{y}{ }^{+} h_{y}{ }^{*}+\alpha_{3} y_{z}{ }^{+} h_{z}{ }^{*} \\
& y^{2}+z^{2}=x^{2}+\alpha_{1}^{2}\left(1-y_{x}^{+2}-h_{x}^{* 2}\right)+ \\
& \alpha_{2}^{2}\left(1-y_{y}{ }^{+2}-h_{y}^{* 2}\right)+\alpha_{3}^{2}\left(1-y_{z}^{+2}-h_{z}^{* 2}\right)+ \\
& 2 \alpha_{1} \alpha_{2} y_{z}{ }^{+} h_{z}{ }^{*}+2 \alpha_{1} \alpha_{3} y_{y}{ }^{+} h_{y}{ }^{*}+2 \alpha_{2} \alpha_{3} y_{x}{ }^{+} h_{x}{ }^{*} \\
& P_{h}(\vec{y})=N(A, B, C) \exp (x) \mathrm{I}_{0}\left(\sqrt{y^{2}+z^{2}}\right) \\
& N(A, B, C)^{-1}=\frac{1}{2} \int_{-1}^{1} d t \exp \left(\alpha_{3} t\right) \mathrm{I}_{0}\left(\frac{A^{2}-B^{2}}{2}(1-t)\right) \mathrm{I}_{0}\left(\frac{C^{2}}{2}(1+t)\right)
\end{aligned}
$$

By variation of the position of the maximum in Euler-space, $g_{0}$, of the ellipsoid's principal axes $a, b, c$ (corresponding to the components half widths) and the orientation of the principal axes with respect to the sample coordinate system, $g_{d}$, the model function can be made to fit a variety of texture component types ranging from isotropic components over incomplete fibres to complete fibre components and even more general shapes. In figure 2, some examples for the influence of the parameters on the shape of the model function are given.

With the help of the model function (3) an ODF can be represented as a weighted sum of a small number of anisotropic texture components. The procedure of finding starting values for model parameters and of parameter refinement is described in (Helming, Eschner, 1990) and (Eschner, 1994).

The result of a texture analysis by means of anisotropic texture components are the positions of the components in Euler-space, the corresponding volume parts (weighting factors) and quantitative information about the scattering of crystallite orientations around the maximum positions, given in the form of half width parameters and fibre axes. It is worth noting that the recalculated ODF is conditionally ghost corrected (Matthies, Vinel, Helming, 1987). 


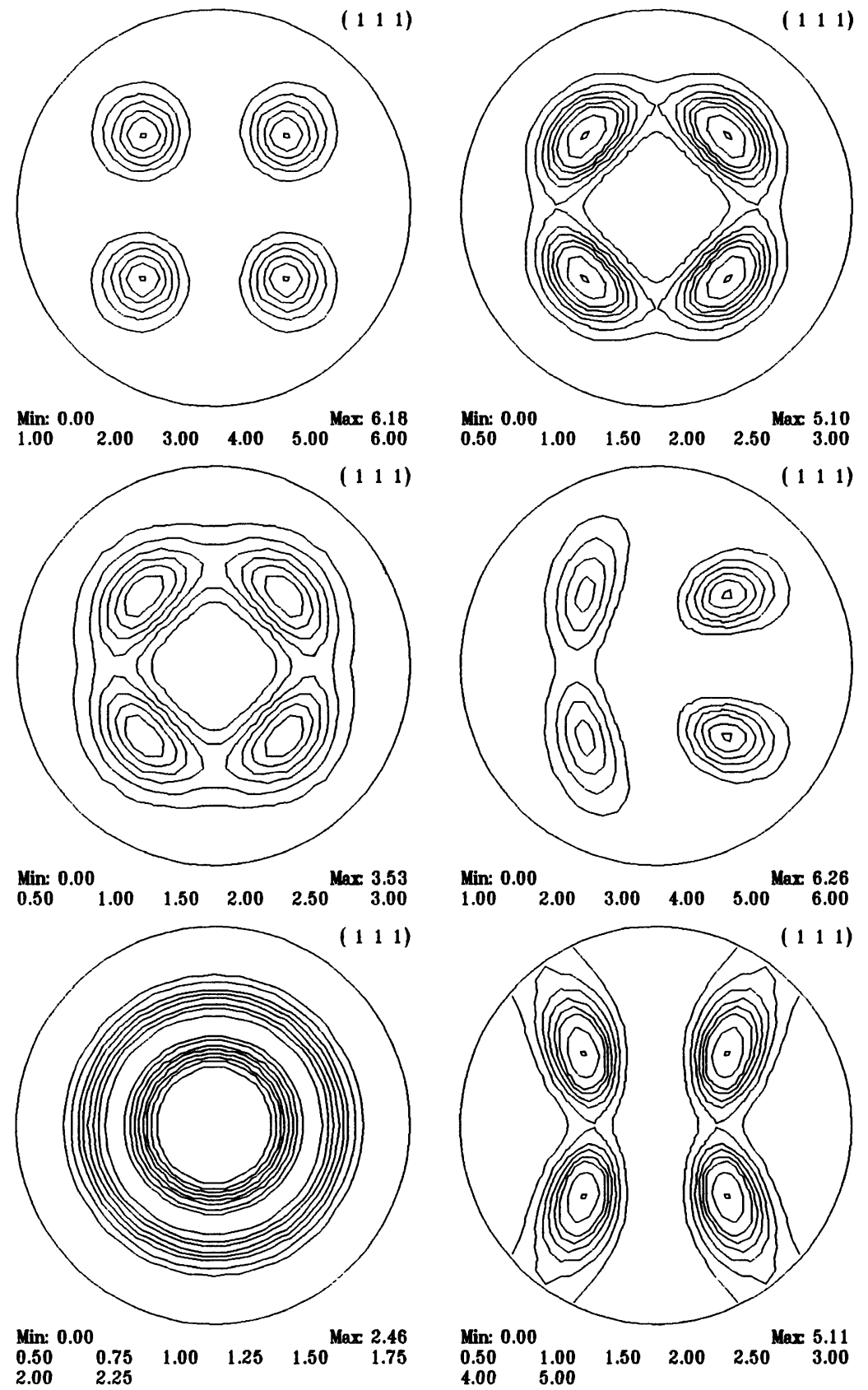

Figure 2 Examples for the influence of parameter variation on the shape of anisotropic texture components. Left column: Transition from a spherical component (ellipsoid degenerated into sphere) to a complete fibre (ellipsoid degenerated into equiaxed disc of zero thickness). Right column: Rotation of incomplete fibre from the $z$-direction (perpendicular to the drawing plane) to the $x$-direction of the sample coordinate system (pointing to the right). 


\section{EXPERIMENTAL PROCEDURE}

In this study, the texture changes of aluminum subject to tensile testing were investigated. A commercial aluminum alloy AA5182, supplied by Hoogovens Aluminium, Belgium, was used. The material contained approximately $4 \% \mathrm{Mg}$; its chemical composition is given in table 1.

Standard tensile test samples ( $45 \mathrm{~mm}$ long, $10 \times 4 \mathrm{~mm}^{2}$ cross section) were prepared and deformed in a tensile test up to elongations as shown in table 2 at a strain rate of $1 \mathrm{~mm} / \mathrm{min}$. During the test, serrated yielding was observed.

For the initial state and for each deformed sample four incomplete pole figures ((111), (200), (220) and (311)) were measured and analyzed, both by decomposition into anisotropic texture components and the iterative series expansion method. The measurement was carried out on the surface of the samples having been electrolytically polished before testing. Co-K $\alpha$-radiation was used. The pole figures were recorded in steps of $2^{\circ}$ of the tilting angle from $1^{\circ}$ up to a maximum tilt of $79^{\circ}$. During the measurement a translational $(6 \mathrm{~mm})$ and rotational $\left(5^{\circ}\right)$ integration movement of the sample was carried out.

In figure 3, the microstructure of the initial state is shown. It is characterized by nearly equiaxed grains with a mean grain size (mean chord length) of approximately $15 \mu \mathrm{m}$. In figure 4 , pole figures of the undeformed sample are shown. The starting texture is characterized by an anisotropic cube texture component; the presence of an additional component is not obvious.

\section{RESULTS}

\section{Experimental Data}

Figures 5 and 6 illustrate the texture changes in aluminum AA5182 during tensile testing by the corresponding changes of the (111) and (200) pole figures. The direction

Table 1 Chemical composition of aluminum alloy AA5182 used in tensile tests.

\begin{tabular}{lcccc}
\hline $\mathrm{Mg}$ & $\mathrm{Fe}$ & $\mathrm{Mn}$ & $\mathrm{Si}$ & others \\
\hline $4.3 \%$ & $0.3 \%$ & $0.3 \%$ & $0.2 \%$ & $<0.05 \%$ \\
\hline
\end{tabular}

Table 2 Elongation $\varepsilon$ and true deformation $e$ of samples deformed in tensile testing.

\begin{tabular}{lcc}
\hline Sample & Elongation $\varepsilon, \%$ & Deformation $e$ \\
\hline alch 1 & 0 & 0 \\
alt 1 & 2.9 & 0.030 \\
alt 3 & 9.0 & 0.086 \\
alt 5 & 15.9 & 0.148 \\
alt 8 & 21.7 & 0.196 \\
alt 10 & 23.6 & 0.211 \\
\hline
\end{tabular}




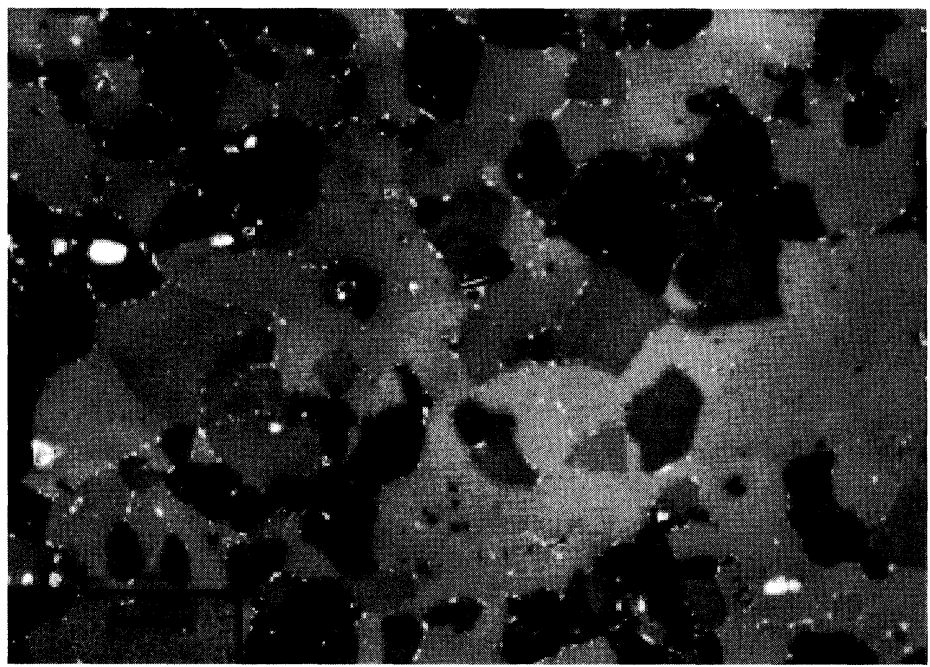

Figure 3 Microstructure of the initial state of aluminum AA5182, $-20 \mu \mathrm{m}$.
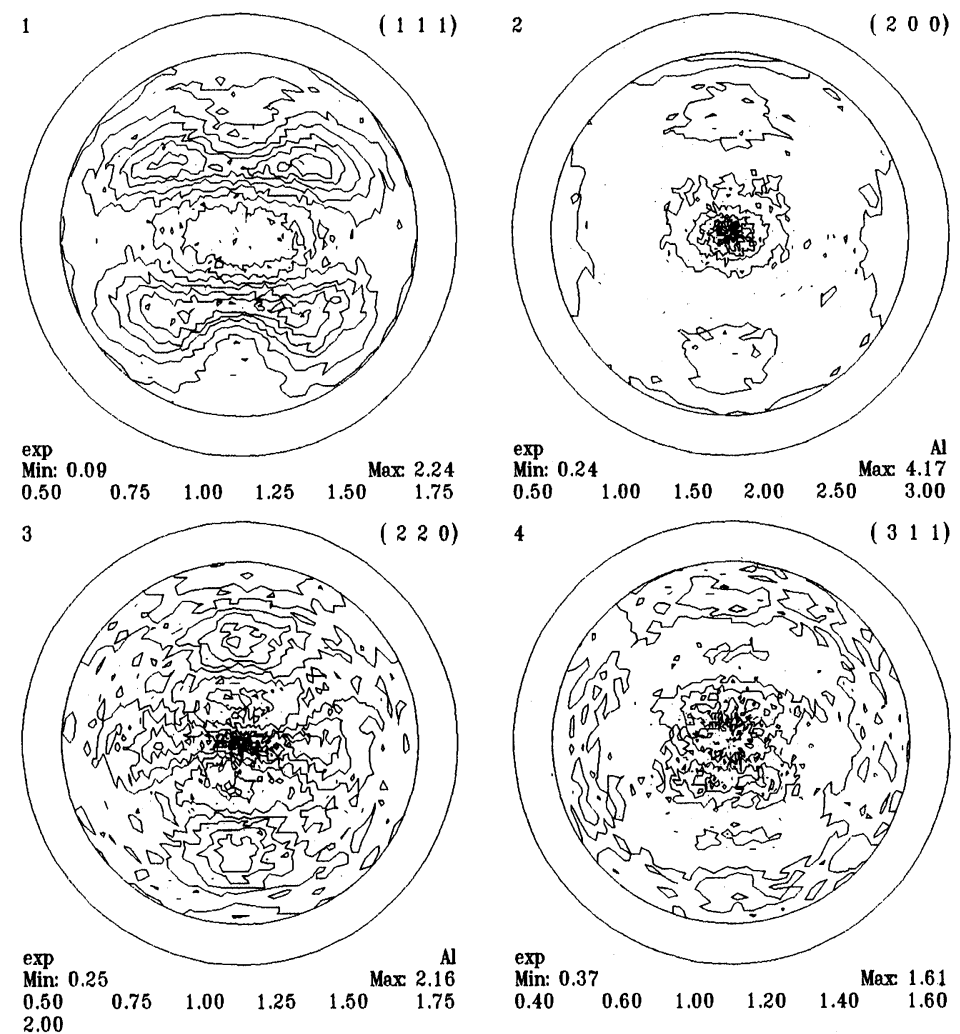

Figure 4 Texture of undeformed material. 

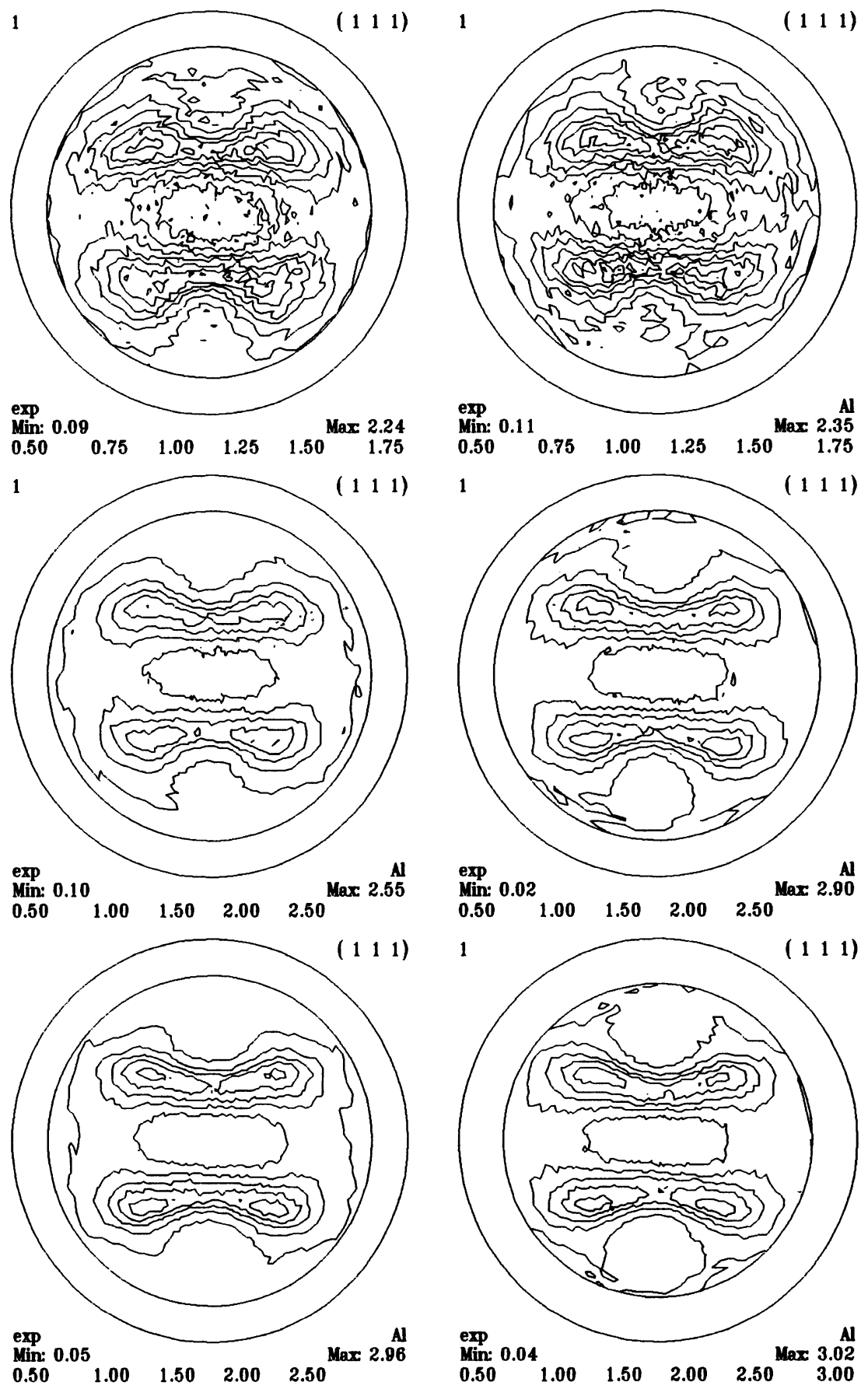

Figure 5 Texture development during tensile testing reported by the changes of (111) pole figures. The direction of testing points to the top of the drawing. 

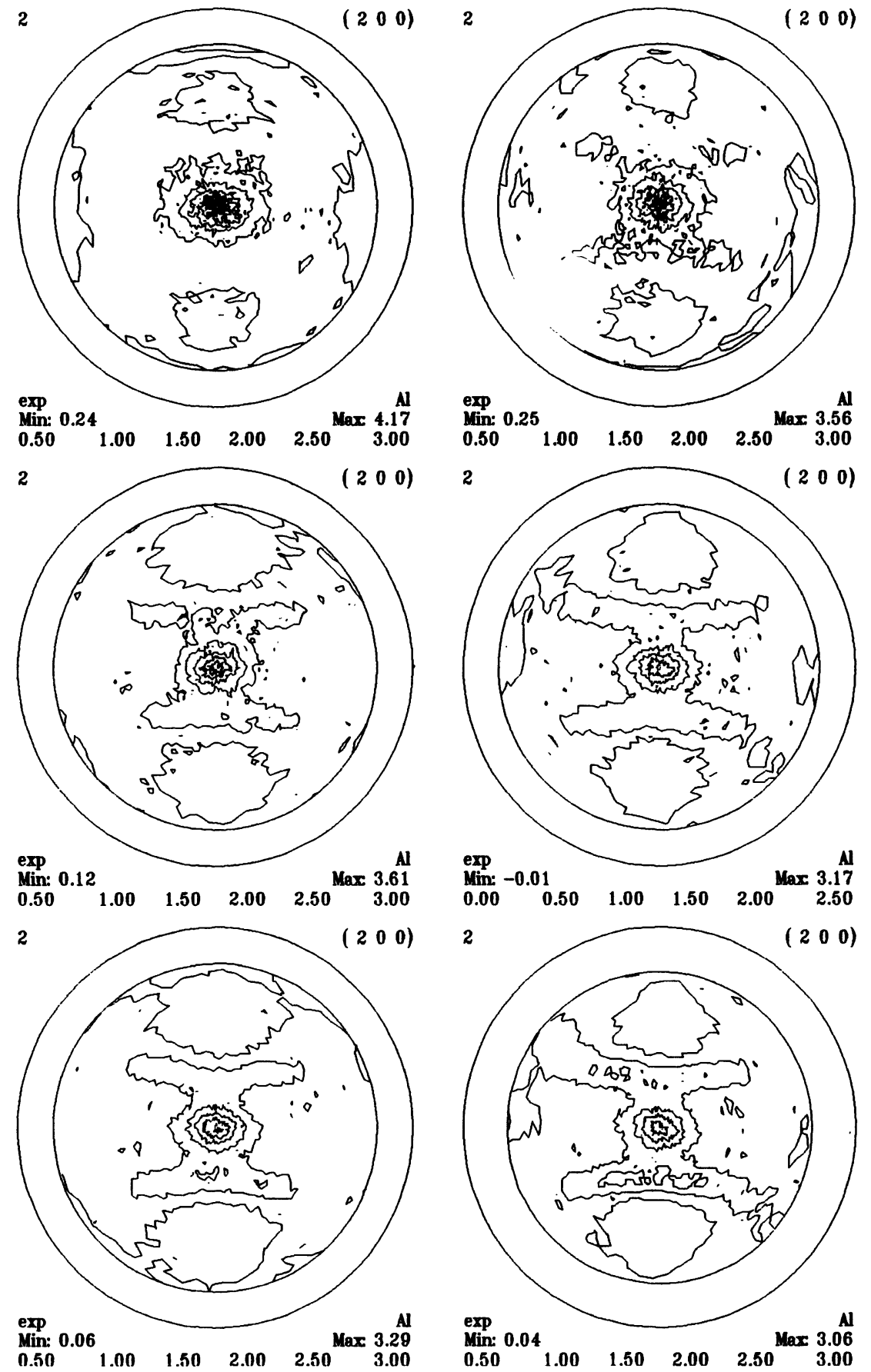

Figure 6 Texture development during tensile testing reported by the changes of (200) pole figures. The direction of testing points to the top of the drawing. 
of tensile testing points to the top. As a result of deformation a slight sharpening of the cube position can be observed, which is attended by the formation of two symmetrically equivalent texture components near to the brass position. The overall change of texture is weak due to the rather small deformation degree.

The sample symmetry can be considered to be orthorhombic; some deviations observed, e.g. in the (200) pole figures, were neglected.

\section{ODF Analysis}

\section{Texture decomposition}

In figure 7, the decomposition of the texture into anisotropic components is demonstrated for the highest deformation degree.

As with all deformation degrees, the experimental pole figures can well be approximated by a superposition of a brass-like and a cube position. Both texture components exhibit a fibre-type character with a fibre axis along the direction of tensile testing, the cube component being closer to an isotropic distribution.

\section{Texture evolution}

As a result of the texture analysis by means of anisotropic texture components, the textures under investigation are completely represented by the parameters of a small number of components: by the volume fractions of components, by their half widths (calculated from the principal axis dimensions of the corresponding ellipsoids), by their positions in Euler-space and by the orientations of the ellipsoids with respect to the sample coordinate system. Texture changes can be represented by the corresponding changes of texture component parameters.

Figures 8 to 10 as well as figure 11 show the texture changes in aluminum during tensile testing in terms of changes of the texture component parameters.

As can be seen from figure 8 , the volume fractions of brass-like and cube components remain nearly unchanged during the deformation. In addition to the volume fraction of the cube component, determined by texture decomposition, equivalent volume fractions were calculated by integrating the ODF, determined by means of the iterative series expansion method, within a sphere of $40^{\circ}$ diameter around the cube position (Zuo, 1992). Because this diameter corresponds approximately to the full width at half of maximum of the cube component (refer to figure 9), ODF integration within this sphere yields $87 \%$ of the volume fraction of the cube component, if a Gaussian shape is assumed (Matthies, Vinel, Helming, 1987). Taking into account such a relation between the volume fractions determined by texture decomposition and ODF integration respectively, the agreement of the data shown in figure 8 is rather good.

In figure 9, the three half widths of the cube component calculated from the principal axis lengths of the corresponding ellipsoid are shown. It can be seen that the cube component sharpens by decrease of half widths, the smallest one remaining practically constant. The difference between large and small half widths decreases, i.e. the cube component is becoming more isotropic during deformation.

In contrast to the cube component, the largest half width of the brass-like position, as shown in figure 10, increases or at least remains constant with increasing deformation. The smaller half widths, on the other hand, decrease by approximately the same amount, i.e. the brass-like component tends to form a fibre.

In figure 11 for all deformation degrees, the most probable crystallite orientations for the cube and brass-like components (left row) and the orientation of the 
1
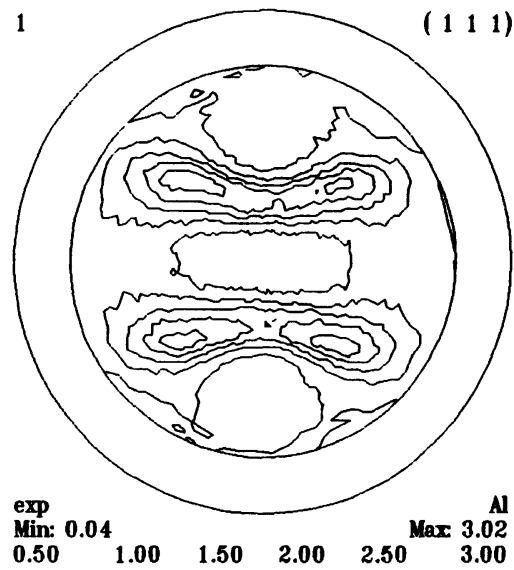

1
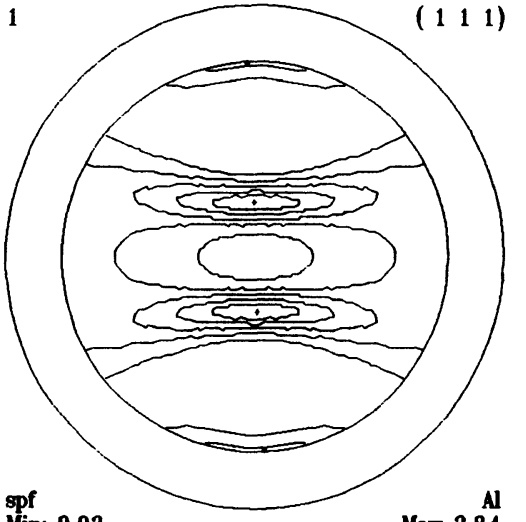

spf 0.03

$\begin{array}{lllll}0.50 & 1.00 & 1.50 & 2.00 & 2.50\end{array}$

1

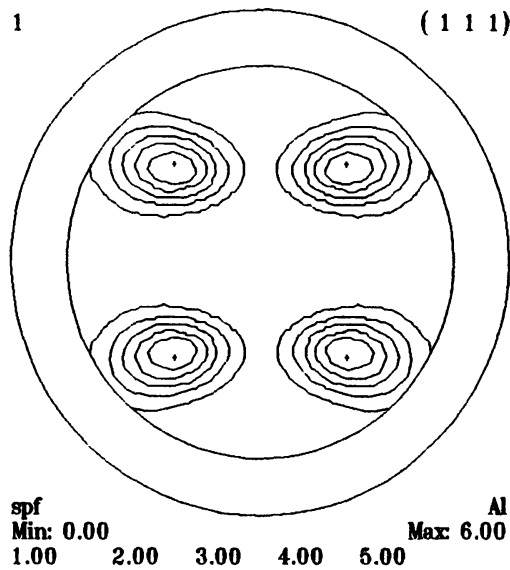

2
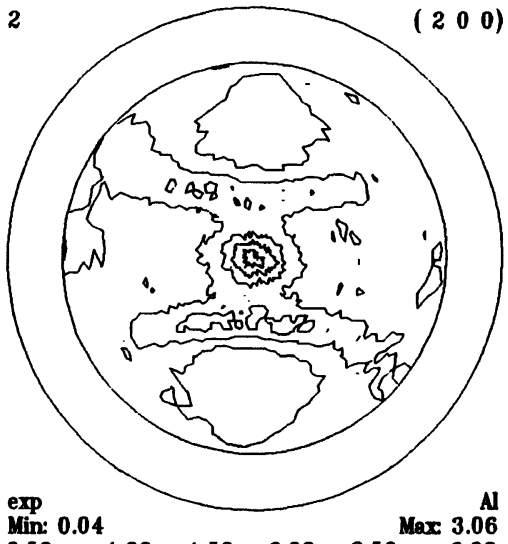

$\begin{array}{llllll}0.50 & 1.00 & 1.50 & 2.00 & 2.50 & 3.00\end{array}$

2

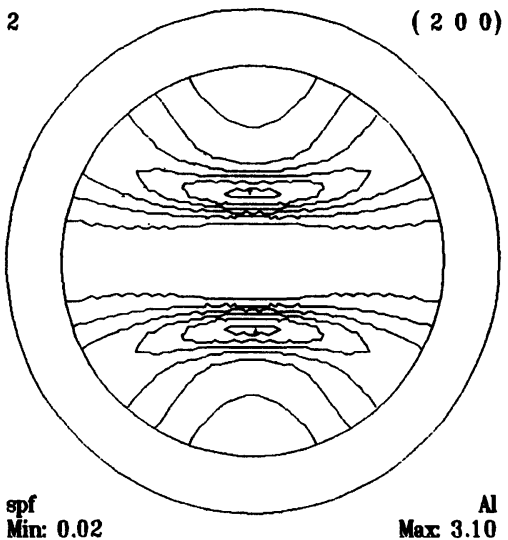

$\begin{array}{llllll}0.50 & 1.00 & 1.50 & 2.00 & 2.50 & 3.00\end{array}$

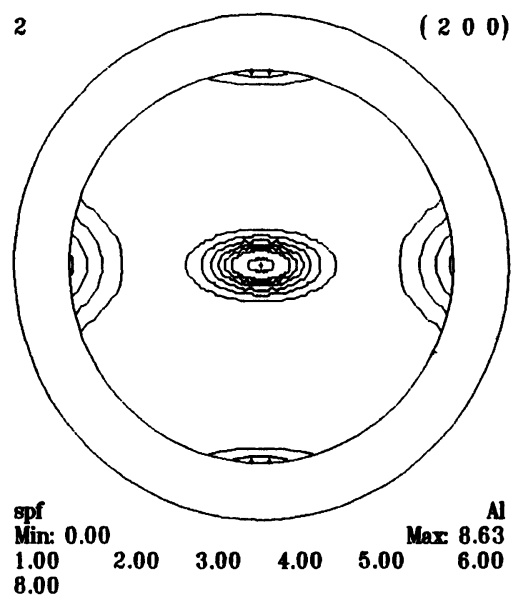

Figure 7 Decomposition of experimental (111) and (200) pole figures into anisotropic texture components: first row - experiment, second row - two symmetrically equivalent components near the brass position, third row - component at cube position. The elongation of the sample is $23.6 \%$ 


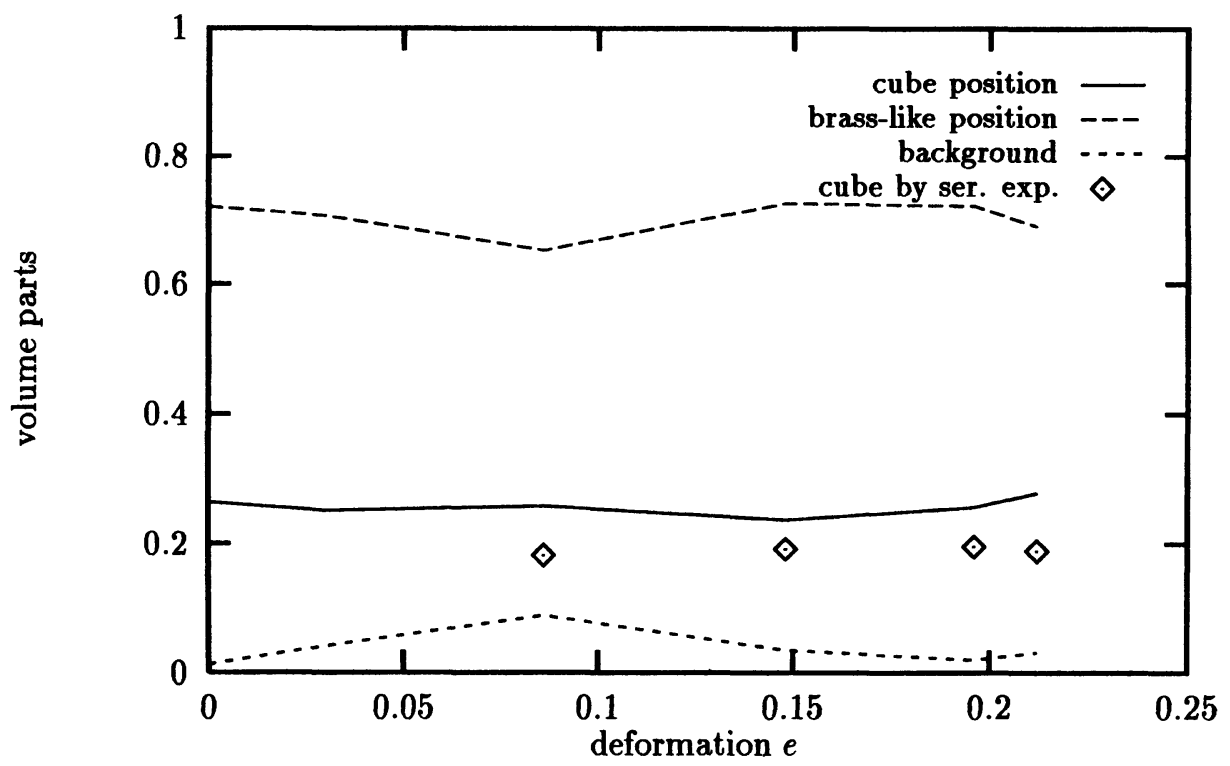

Figure 8 Dependence of volume parts of cube and brass-like texture components on the deformation e during tensile testing. Large half widths correspond to the direction of tensile testing, small ones to the transverse direction of the sample.

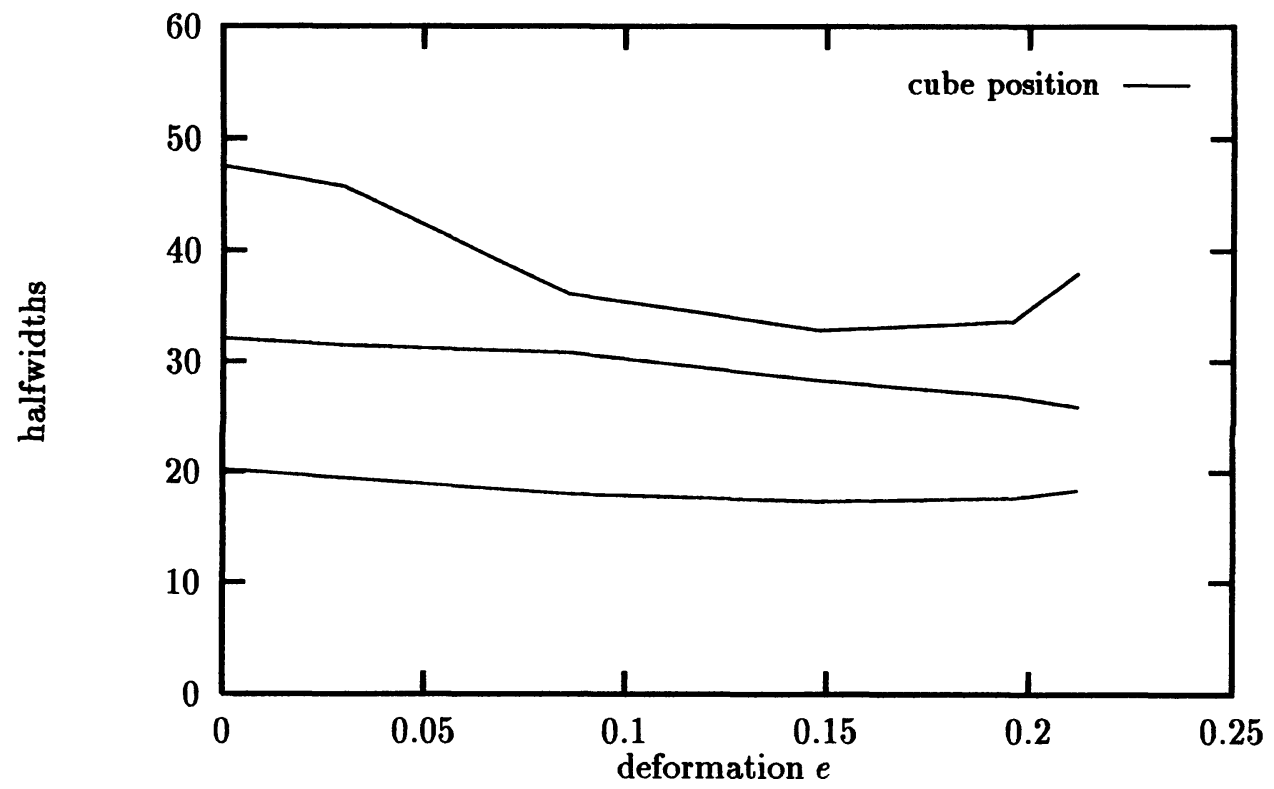

Figure 9 Dependence of half widths of the cube texture component on the deformation $e$ during tensile testing. Large half widths correspond to the direction of tensile testing, small ones to the transverse direction of the sample. 


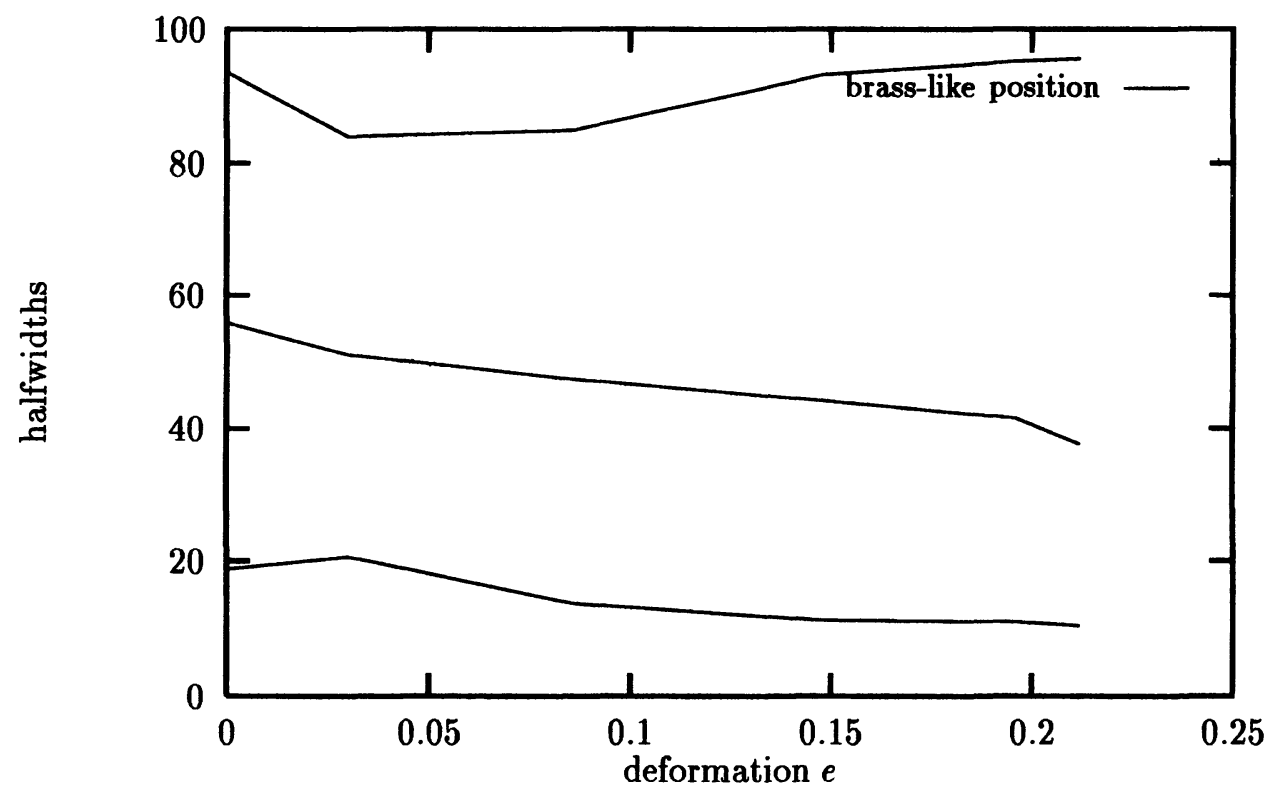

Figure 10 Dependence of half widths of the brass-like texture component on the deformation $e$ during tensile testing. Large half widths correspond to the direction of tensile testing, small ones to the transverse direction of the sample.

corresponding ellipsoids describing crystallite orientation scattering (right row) are shown. The orientations are visualized by triangles on the upper half of the unity sphere, which are formed by three directions perpendicular to each other: by (200) crystal directions in the case of crystallite orientations and by the principal axes in the case of ellipsoid orientations. As for pole figures shown in previous figures, the direction of tensile testing points to the top of the drawing.

As can be seen from figure 11, the most probable crystallite orientations of the cube component can be reproduced for all deformations with high accuracy. The corresponding orientations of the brass-like component exhibit some scattering around the normal direction of the samples. When represented in terms of Miller indices, orientations ranging from $\{110\}<113>$ to $\{110\}<223>$ are found; see also (Wassermann, Grewen, 1962).

For both texture components, the principal axes of the ellipsoids are near to the axes of the sample coordinate system. The scattering of the orientation of the ellipsoids is higher, compared to the crystallite orientations.

In all cases, i.e. for all components and all deformation degrees, the largest half width corresponds to the direction of tensile testing and the lowest half width to the transverse direction of the sample.

\section{Accuracy}

A texture description in terms of e.g. two anisotropic components has 21 parameters, i.e. 10 Parameters for each component (a volume fraction, three Euler angles for the center of the distribution, three Euler angles for the orientation of the ellipsoid and 


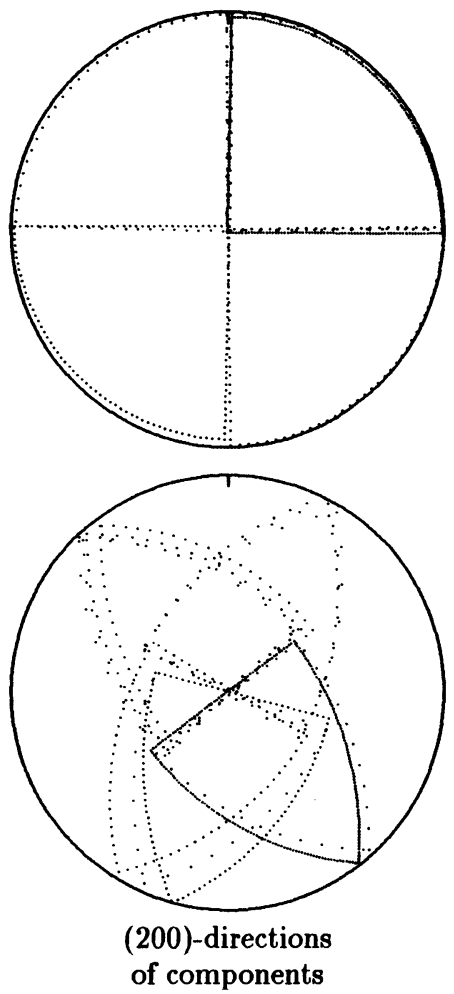

cube position
$\{001\}\langle 100\rangle$

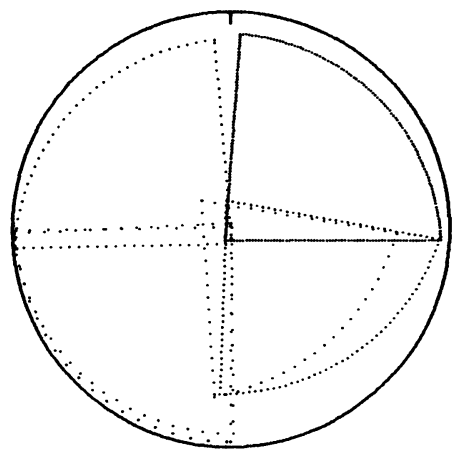

brass-like position

$\{110\}\langle 113\rangle \ldots$

$\{110\}\langle 223\rangle$

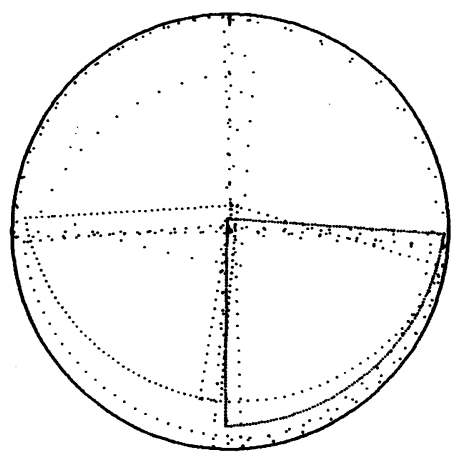

principal axes of ellipsoids

Figure 11 The orientation of crystallites and ellipsoids. Left row: the most probable crystallite orientations are visualized by triangles on the upper half of a unity sphere formed by (200) crystal directions. Right row: the orientations of ellipsoids are visualized by triagles formed by the principal axes of the ellipsoids.

three principal axis lengths) and 1 parameter for the isotropic texture background. If expanded into a series, for the representation of an ODF typically no less than 125 parameters are required (at an expansion degree of 22 and cubic-orthorhombic symmetry). It is not obvious that 21 parameters are sufficient for an adequate ODF representation.

As a rule, the accuracy of an ODF calculation (and also of the experiment) is determined by the deviations between experimental and recalculated pole figures, which can quantitatively be characterized by the so-called $\operatorname{RP}(\varepsilon)$-values (Matthies, Vinel, Helming, 1987). They are defined as the mean relative error of normalized pole figure values greater than the threshold $\varepsilon$.

For all samples, the experimental pole figures were compared with recalculated pole figures from a series expansion and from a decomposition into anisotropic components. The corresponding $\mathrm{RP}(0.01)$ - and $\mathrm{RP}(1)$-values were calculated and compiled into table 3.

In most cases, the accuracy of the series expansion method and of the component method are comparable. As can be seen from the RP(0.01)-values, low pole figure values were recalculated somewhat better by the series expansion method, which is due to 
Table 3 Agreement between experimental and recalculated pole figures, characterized by RP-values, for two pole figure inversion methods: the series expansion method and the component method. Ranges cover minimum and maximum RP-values calculated for four pole figures.

\begin{tabular}{lcccc}
\hline & \multicolumn{2}{c}{$R P(0.01), \%$} & \multicolumn{2}{c}{$R P(1), \%$} \\
& ser. $\operatorname{exp.}$ & comp. & ser. exp. & comp. \\
\hline alch 1 & $8-13$ & & $7-9$ \\
alt 1 & & $12-15$ & & $7-10$ \\
alt 3 & $13-29$ & $17-28$ & $7-15$ & $8-12$ \\
alt 5 & $13-18$ & $14-27$ & $5-11$ & $8-9$ \\
alt 8 & $13-36$ & $20-36$ & $7-14$ & $8-12$ \\
alt 10 & $13-19$ & $14-26$ & $6-11$ & $8-12$ \\
\hline
\end{tabular}

the least-squares approach used in the component method (Helming, Eschner, 1990). Accordingly, higher pole figure values were better recalculated by decomposition into anisotropic texture components.

\section{CONCLUSIONS}

The decomposition of experimental pole figures into anisotropic texture components leads to a texture representation by a very small number of parameters, each having an obvious meaning and a well defined influence on the texture representation. This makes it easier to track texture changes during various steps of material treatment or to relate texture data to other measurements.

For the example of a commercial aluminum alloy deformed in tensile tests, the kind of information that can be obtained by means of anisotropic texture components was demonstrated. It was shown that, despite of the small number of parameters, the accuracy of the texture representation by anisotropic components is comparable to the accuracy achieved by the series expansion method.

\section{References}

Bunge, H.-J. (1969). Mathematische Methoden der Texturanalyse, Akademie-Verlag, Berlin 1969.

Dnieprenko, V. N. and Divinskii, S. V. (1993). A New Approach to Describing Three-Dimensional Orientation Distribution Functions in Textured Materials, I. Formation of Pole Density Distribution on Model Pole Figures, Textures \& Microstructures, 22 (1993), 73-85.

Eschner, Th. (1993). Texture Investigation by Means of Model Functions, Mat. Sci. Forum, 133-136 (1993), 139-144.

Eschner, T. (1994). Quantitative texture analysis by decomposition of diffraction pole figures into texture components (in German), thesis, Freiberg University of Mining and Technology, Germany, 1994.

Helming, K. and Eschner, T. (1990), A New Approach to Texture Analysis of Multiphase Materials using a Texture Component Model, Cryst. Res. Technol., 25 (1990), K203-K208.

Matthies, S. (1980). Standard Functions in Texture Analysis, phys. stat. sol. (b), 101 (1980), K111-K115.

Matthies, S., Vinel, G. W. and Helming, K. (1987). Standard Distributions in Texture Analysis, Vol. 1, Akademie-Verlag, Berlin 1987. 
Savyolova, T. I. (1987). Preface of: Novye Metody Teksturnogo Analisa (in Russian), Metallurgia, Moskva 1987.

Wassermann, G. and Grewen, J. (1962). Texturen metallischer Werkstoffe, Springer-Verlag, Berlin Göttingen Heidelberg 1962.

Zuo, L., Muller, J. and Esling, C. (1993). Volume Fractions of Texture Components in Polycrystalline Materials, J. Appl. Cryst., 26 (1993), 422-425. 